\title{
Prevalence of Malaria and Genetic Diversity and Antibody Response to Malaria Vaccine Candidate (Eba-175) in Children in Ngali and Mfou, Central Region of Cameroon
}

\author{
Azua Ekokobe Wilfred ${ }^{1}$, Nkengafac Nyiawung Fobellah ${ }^{2 *}$, \\ Omeichu Agwenam Amadeus ${ }^{3}$, Rose Leke ${ }^{4}$, Mbatcham Wilfred ${ }^{5}$ \\ and Jude Bigoga ${ }^{6}$ \\ ${ }^{1}$ Faculty of Medical and Biomedical science, University of Yaounde I, Cameroon \\ ${ }^{2}$ Lecturer, School of health and Human Sciences, Saint Monica University Higher \\ Institute of Buea, Cameroon \\ ${ }^{3}$ Faculty of Health Sciences, University of Buea, Cameroon \\ ${ }^{4}$ Professor, Malariologist and Emeritus Professor of Immunology, Faculty of \\ Medical and Biomedical Science, University of Yaounde I, Cameroon \\ ${ }^{5}$ Professor of Biotechnology Laboratory, Faculty of Medical and Biomedical science, \\ University of Yaounde I, Cameroon \\ ${ }^{6}$ Professor, Faculty of Medical and Biomedical Science, University of Yaounde I, \\ Cameroon \\ *Corresponding Author: Nkengafac Nyiawung Fobellah, Lecturer, School of health \\ and Human Sciences, Saint Monica University Higher Institute of Buea, Cameroon.
}

Received: February 16, 2021

Published: March 05, 2021

(C) All rights are reserved by Nkengafac

Nyiawung Fobellah., et al.

\begin{abstract}
Introduction: Plasmodium falciparum (P. $f$ ) malaria is a major public health challenge and a serious impediment to socioeconomic development. Despite preventive and curative measures, malaria still remains a major problem in sub-Saharan Africa. To be effective, a multifaceted malaria elimination program should include a vaccination program. Several $P$. $f$ stage-specific antigens have been characterized as vaccine candidates through molecular epidemiology, among these is the EBA-175 antigen. Many studies have been done on the immune responses and also on the genetic diversity of EBA-175, but little is understood about the possible relationship between the genetic diversity and immune response. Understanding the association that might exist between the genetic diversity and the antibody response to EBA-175 is crucial in broadening our knowledge of the immune-epidemiology of this candidate vaccine and also in the production of a future multivalent vaccine. Thus we sought to determine the prevalence of malaria among children and the sociodemographic characteristics of these Children across the Ngali and Mfou localities.

Methodology: We carried out an Analytical cross-sectional study from November 2016 to May 2017 at the Biotechnological research center in Yaoundé. We included in our study children aged 1 year to 15 years, diagnosed with P.f, and whose parents or legal guardians gave informed consent. Venous Blood samples were collected in both localities, then analyzed using nested PCR and multianalyte platform technology (Luminex 100) for data on gene diversity and antibody level responses respectively. Data was analysed using graph-pad prism. All p values below 0.05 were considered as significant.

Results: In total, 320 participants between the ages of 1 year and 15 years were recruited for this study. The mean age was 6.9 years. we found an overall prevalence of malaria, of 76\%; 80\% in Ngali and 72\% in Mfou. The 6-10 years age had the highest prevalence of
\end{abstract}

Citation: Nkengafac Nyiawung Fobellah., et al. "Prevalence of Malaria and Genetic Diversity and Antibody Response to Malaria Vaccine Candidate (Eba-175) in Children in Ngali and Mfou, Central Region of Cameroon". Acta Scientific Medical Sciences 5.4 (2021): 14-17. 
Prevalence of Malaria and Genetic Diversity and Antibody Response to Malaria Vaccine Candidate (Eba-175) in Children in Ngali and Mfou, Central Region of Cameroon

malaria 0f 44.7\%. The main EBA-175 genotypes found in our study population were F loop, C loop and 400bp. With F loop being the most predominant allele form.

Conclusion: This study depicts a high prevalence of malaria in the Ngali and Mfou rural communities and was more common in males. The F loop is more predominant in both localities.

Keywords: Malaria; Genetic Diversity; Plasmodium falciparum

\section{Introduction}

Plasmodium falciparum malaria is a major public health challenge and a serious impediment to socioeconomic development, particularly in sub-Saharan Africa (SSA), where $85 \%$ of the global burden occurs. According to the latest WHO estimates, released in December 2016, there were 212 million cases of malaria in 2015 and 429000 deaths. Most of these deaths occurred in the African Region (92\%), followed by the South-East Asia Region (6\%) and the Eastern Mediterranean Region (2\%) [1]. Efforts have been made over the years to fight against the burden of this disease and these include preventive measures (mainly by long-lasting insecticide impregnated mosquito nets, insecticide sprays, Intermittent Preventive Treatment (IPT) and Seasonal Malaria Chemoprevention (SMC)) and Curative Measures (mainly by Artermesine Combined therapy (ACT) for simple malaria and injectable doses of the antimalaria drugs for severe forms of the disease) [2]. Many studies have been done on the immune responses and also on the genetic diversity of EBA-175, but little is understood about the possible relationship between the genetic diversity and immune response [3].

Despite these measures, malaria still remains a major problem in sub-Saharan Africa because the Female anopheles mosquito is also evolving and developing resistance to insecticides. Furthermore, there is the development of anti-malarial drug resistance, primarily against those drugs targeting the parasite asexual erythrocytic stages [4]. To be effective, a multifaceted malaria eradication program should include vaccination and effective control of mosquito vectors [5]. Moreover, its prevalence and mortality still poses a problem especially in SSA.

\section{Materials and Methods}

\section{Study design and setting}

The study was an analytical cross- sectional study carried out at the malaria research lab of the Biotechnological Center of Uni- versity of Yaounde 1 of the Center Region of Cameroon. The study was carried out from November 2016 to May 2017. The BTC runs projects in human immune responses in disease endemic countries, and hosts the International Centre for Development Research that investigates pregnancy-associated and neonatal immunity in Malaria (co-funded by NIH and WHO) as well as plant and animal biotechnology.

\section{Study population and sampling}

The study population included Children at the localities of Ngali, and Mfou between ages of 1year and 15 years. We included Children diagnosed with Plasmodium falciparum in the blood and all Children whose parents or Guardians signed the consent form. Meanwhile, we excluded Immuno-comprised Children. The minimum sample was calculated using the formula of Lorentz [6]. This calculations led to a sample size of 320 .

\section{Study procedure}

After obtaining ethical approval from the Ethical Committee of the Faculty of Medicine and Biomedical Sciences, University of Yaoundé I, to carry out this study, the authorization of the directors of the different laboratories were obtained. Also, copies of the protocol were distributed to the above personalities. We worked at the field, the Laboratory and analyzied the results after. At the Field, Prints signed consent forms, we recruited participants and collected and stored samples gotten from participants.

All data was entered into Microsoft Excel 2010 and validated.

Pie or bar charts were used to demonstrate the distribution of discrete quantitative data while histograms were used for continuous data. A P-value $<0.05$ was considered to be statistically significant. 


\section{Results}

Sociodemographic characteristics of participants

A total of 320 participants were included in this study, of which 150 were females $(46.8 \%)$. In the locality of Ngali we had a total of 140 participants, with more females 65 (46.5\%) and 75 males (53.5\%). Meanwhile in Mfou, we had a total of 180 participants, 85 females (47.2\%) and 95 males (52.8\%). See table 1 below. The participants were distributed into three groups according to their age. The overall mean age was 7.8 in the locality of Ngali we had a mean of 8.8 years while in Mfou we had a mean of 6.9 years as shown on table 2 below.

\begin{tabular}{|l|c|c|}
\hline \multicolumn{1}{|c|}{ Sex } & $\begin{array}{c}\text { NGALI } \\
(\mathbf{N = 1 4 0 )}\end{array}$ & $\begin{array}{c}\text { MFOU } \\
(\mathbf{N = 1 8 0})\end{array}$ \\
\hline & Percentage & Percentage \\
\hline Male & $53.5 \%(75 / 140)$ & $52.8 \%(95 / 180)$ \\
\hline Female & $46.5 \%(65 / 140)$ & $47.2 \%(85 / 180)$ \\
\hline
\end{tabular}

Table 1: Distribution by Gender per site.

\begin{tabular}{|l|c|c|c|}
\hline $\begin{array}{c}\text { Age of } \\
\text { the child } \\
\text { (years) }\end{array}$ & $\begin{array}{c}\text { NGALI } \\
(\mathbf{N}=\mathbf{1 4 0})\end{array}$ & $\begin{array}{c}\text { MFOU } \\
(\mathbf{N}=\mathbf{1 8 0})\end{array}$ & $\begin{array}{c}\text { OVERALL } \\
(\mathbf{N}=\mathbf{3 2 0})\end{array}$ \\
\hline $1-5$ & $27.8 \%(13 / 140)$ & $42.2 \%(76 / 180)$ & $27.8 \%$ \\
\hline $6-10$ & $44.7 \%(82 / 140)$ & $33.8 \%(61 / 180)$ & $44.7 \%$ \\
\hline $11-15$ & $27.5 \%(45 / 140)$ & $24 \%(43 / 180)$ & $27.5 \%$ \\
\hline
\end{tabular}

Table 2: Distribution of study population by Age.

\section{Prevalence of malaria in Mfou and Ngali \\ Global prevalence}

In this study, $24 \%$ of children were diagnosed malaria negative while $76 \%$ were diagnosed malaria positive yielding a malaria prevalence of $76 \%$ (Figure 1).

\section{Discussion}

Malaria elimination has been part of the Global Technical Strategy for Malaria goals. The prevalence of malaria is still on a rise. This goal has been fettered by the development of resistance to drugs and insecticides, hence recent colloquies on malaria elimination focus on the development of an effective P. falciparum vaccine.

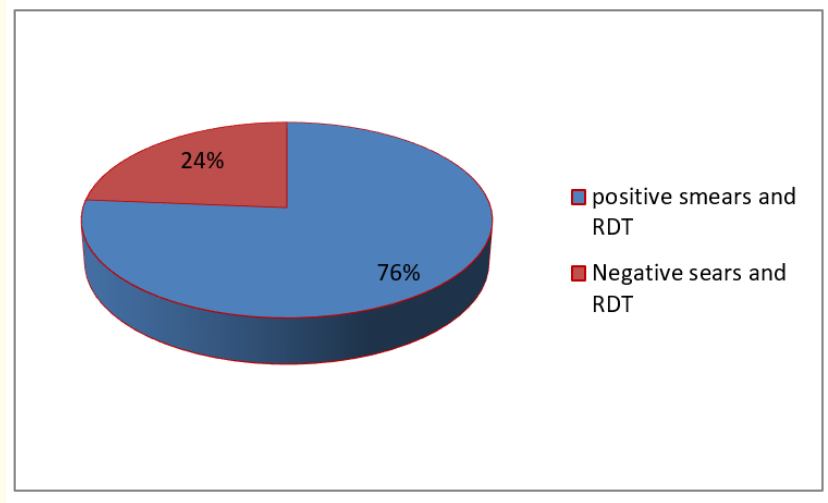

Figure 1: Global prevalence.

P. falciparum has a long evolutionary history with its human host and exhibits extensive genetic diversity, particularly in the surface antigens that have been under selective pressure by the human immune response and that have been the main targets of subunit vaccines. Polymorphism may enable parasites to evade immune responses elicited by past exposure to diverse forms of the same antigen. Hence extensive polymorphism in many vaccine candidate proteins may limit the efficacy of vaccines based on just a few allelic variants that may not be broadly cross-protective against diverse antigens in natural infections. This could lead to vaccine-resistant malaria with non-vaccine type parasites growing in frequency within vaccinated populations. Merozoite antigens are important targets of acquired immunity and thus have significant potential as vaccine candidates.

We had a global malaria prevalence of $76 \%$; a malaria prevalence of $80.1 \%$ at Ngali and $72 \%$ at Mfou. The prevalence at Mfou was slightly lower than that recorded in 2015 of 77\% by Francis Z., et al. [7] in 2015 among school children in the same area. This high prevalence could be due to the fact that both studies were carried out at the same period during the wet season. In addition to these findings, our age group with the highest prevalence was 6-10 years age group. This finding was similar to that found in India in 2012 and that found in Cameroon by (Soulama., et al. [8]), this lug in the trend of the highest prevalence from 0-5 years to 6-10 years can be explained by the increased measures employed on the critical 
age group of 0-5years which has in turn limited measures on the other age groups. $P$. falciparum was the main species form in both Ngali and Mfou. Interestingly there was a trend to the association between parasitemia and hemoglobin levels, this relationship was indirect and this can be explained by the cellular lysis and sequestration effects of the malaria parasite on Red blood cells. The indirect relationship between age and parasitemia observed is consistent with the fact that below 5 years of age children have a fragile immune system and depend mostly on maternal antibodies, but as age increases, there is maturity of the immune system and thus an increase in antibody response.

\section{Conclusion}

This study depicts a high prevalence of malaria in the Ngali and Mfou rural communities. The overall prevalence was $76 \%$ and it was more common in males.

\section{Bibliography}

1. WHO | Fact Sheet: World Malaria Report (2016).

2. Which strategies were adopted to prevent and treat malaria? (2017).

3. Ahmed Ismail H., et al. "Subclassresponses and their half-lives for antibodies against EBA175 and PfRh2 in naturally acquired immunity against Plasmodium falciparum malaria". Malaria Journal 13 (2014): 425.

4. WHO | Antimalarial drug resistance (2017).

5. Roberts L., et al. "Malaria. Did they really say ... eradication?" Science 318.5856 (2007): 1544-1545.

6. English M., et al. "Acidosis in severe childhood malaria". Journal of Medicine 90 (1997): 90.

7. Francis Z., et al. "Prevalence of Malaria in central region" (2015)

8. Soulama A. "Allele form on malaria plasmodium parasite" (2012).

\section{Assets from publication with us}

- Prompt Acknowledgement after receiving the article

- Thorough Double blinded peer review

- Rapid Publication

- Issue of Publication Certificate

- High visibility of your Published work

Website: www.actascientific.com/

Submit Article: www.actascientific.com/submission.php

Email us: editor@actascientific.com

Contact us: +919182824667 